\title{
Experiencia en la elaboración de vídeos didácticos por alumnos de Fisiología como parte de su proceso de aprendizaje.
}

\author{
Isabel Sánchez-Vera ${ }^{\mathrm{a}}$, Esther Escudero ${ }^{\mathrm{b}}$, Úrsula Muñoz $^{\mathrm{c}}, \mathbf{M}^{\mathrm{a}}$ José Borrego $^{\mathrm{d}}$ y Rima \\ Barhoum $^{\mathrm{e}}$
}

aisanver@ceu.es, bestheresc@ceu.es, cursula.muñozmoron@ceu.es, $\quad$ d majoborrego@ceu.es, erbarho@ceu.es. Todos los autores tienen la misma procedencia: Sección de Fisiología. Dpto. Ciencias Médicas Básicas. Facultad de Medicina. Universidad San Pablo CEU, Madrid.

\begin{abstract}
The discipline of physiology is challenging to teach. For this reason Physiology I teachers' of Medicine degree are actively involved in updating the teaching methodology used. For this aim, in the last years we have tried to promote the acquisition of different competencies by students such as: increasing their autonomy to monitor their own progress in the subject, searching for knowledge in multiple sources, managing several communication styles and collaborative work to deepen the knowledge and promote its divulgation in the academic environment. For these purposes we have been designer different proposals to the students as the management of engines of computer searches and bibliographic repositories, the creation of didactic videos and the sharing of information and knowledge acquired by students through a computer forum. In this report we have done an analysis and reflection on the acceptance of this methodology among all students of the four groups that taken this academic year 2016-2017.
\end{abstract}

Keywords: didactic vídeos, computer forum, creativity

\section{Resumen}

En la asignatura de Fisiología I del Grado de Medicina los profesores participantes en su impartición están comprometidos de forma activa en actualizar la metodología docente a utilizar. Para ello en los últimos años hemos pretendido fomentar la adquisición de distintas competencias por parte del alumnado como son: el aumento de su autonomía para gestionar su progreso en la asignatura, la búsqueda de conocimientos en fuentes diversas, el manejo de distintas modalidades de comunicación de conocimiento y el trabajo colaborativo para profundizar en la el conocimiento y fomentar su divulgación en el entorno académico. Con esta intención se han ido planteando distintas propuestas a los alumnos como el manejo de motores de búsquedas informáticos y repositorios bibliográficos, realización de vídeos didácticos y puesta en común de la información y conocimientos adquiridos por los alumnos a través de un foro informático. En la presente memoria hemos hecho un análisis y reflexión sobre la aceptación de esta metodología entre todos los alumnos de los cuatro grupos que compone este curso académico 2016-2017. 
Experiencia en la elaboración de vídeos didácticos por alumnos de Fisiología como parte de su proceso de aprendizaje.

Palabras clave: vídeo, foro, creatividad.

\section{Introducción}

En los últimos años los métodos de enseñanza están sufriendo un cambio paulatino con el objetivo de buscar nuevas herramientas con las que los alumnos puedan aprender de una forma más eficiente a la vez que divertida (Arceo et al., 2002; Álvarez et al., 2008). Esta nueva tendencia se está empezando a aplicar también en la enseñanza universitaria de modo que muchos profesores buscan alternativas a las clases tradicionales (Barr at el., 1995). En este contexto también se pretende que los alumnos se sitúen en el centro de las actividades docentes, ya que ellos son los verdaderos protagonistas de su aprendizaje. Además, no hay que olvidar que aunque se utilicen nuevas herramientas didácticas los alumnos deben adquirir las competencias necesarias y que los resultados del aprendizaje deben ser evaluados por el profesor (Molero et al., 2005; Monereo et al., 2003).

En el ámbito de las Ciencias de la Salud el aprendizaje basado en nuevas metodologías no está demasiado desarrollado (Ferro et al., 2009; García, 1993). La mayor parte de las veces a los estudiantes de estas Titulaciones se les enseña a pensar acorde al método científico, pero no se desarrollan suficientemente habilidades como la creatividad o la innovación (Gargallo et al., 2015). En los últimos años los profesores del Área de Fisiología hemos propuesto diferentes metodologías didácticas complementarias a las clases magistrales teóricas y las clases prácticas (Castaño et al., 2007). En nuestra experiencia con los alumnos del Grado en Medicina, tradicionalmente les hacíamos prepararse por su cuenta una parte del temario de nuestra asignatura y exponerlo en clase por grupos mediante presentaciones orales. Aunque se les daban ciertas directrices para llevar a cabo las exposiciones, el resultado eran presentaciones muy largas, pocas veces ajustadas al tiempo disponible y con un contenido excesivo y a veces innecesario. Por ello, las exposiciones resultaban difíciles de seguir por sus compañeros y nuestra impresión es que los alumnos perdían la atención y la motivación, por lo que el aprendizaje no era el adecuado (Machemer et al., 2007). Por otro lado, una de las dificultades con la que nos encontrábamos era poder evaluar de forma objetiva el esfuerzo realizado por los alumnos, ya que se sabe que no todos los alumnos se implicaban por igual, de modo que la carga de trabajo no estaba distribuida de forma equitativa entre todos los miembros del equipo.

En nuestra sociedad, el acceso continuo a la información, completamente digitalizada y audiovisual, permite el uso de los vídeos para reforzar el conocimiento de los alumnos de una forma entretenida a la vez que eficaz (Roman et al., 2007). Mediante la visualización de imágenes se pueden aclarar conceptos de forma más clara y el uso de vídeos en el aula se ha extendido como herramienta didáctica de apoyo para el docente. La elaboración de un vídeo conlleva varias etapas, entre las que destacamos: selección de los contenidos y de los objetivos que se pretenden alcanzar, recoger la documentación para la elaboración del guion, esto es, investigar, buscar bibliografía de fuentes fiables, documentarse, y por último, editarlo con los recursos tecnológicos disponibles (Ferrés et al., 1991). Es decir, la elaboración de un vídeo es un trabajo que hay que planificar y desarrollar y que requiere

(cc)) EY-NC-ND 2017, Universitat Politècnica de València 
tiempo y esfuerzo. Es por ello que pensamos que si los alumnos elaboraban ellos mismos sus propios vídeos, estarían aprendiendo la materia a lo largo de este proceso sin apenas darse cuenta (Ruth at el., 2016).

Por este motivo, con el desarrollo de esta experiencia buscamos por un lado fomentar la creatividad y motivación de los alumnos, haciéndoles partícipes de su propio aprendizaje y por otro lado, valorar de forma real y lo más objetiva posible el grado de implicación de cada alumno en el trabajo realizado fuera del aula.

\section{Objetivos}

El objetivo principal que se plantea es la elaboración por parte de los alumnos de vídeos explicativos de una parte del temario de la asignatura de Fisiología. De este modo se pretende que el diseño y la edición del vídeo les sirva como proceso de aprendizaje, además de poder utilizarse como material didáctico para el resto de sus compañeros.

El objetivo secundario es que el profesor sea capaz de evaluar de forma lo más objetiva posible el trabajo que han realizado fuera del aula.

\section{Desarrollo de la innovación}

La experiencia docente se ha llevado a cabo en la asignatura Fisiología I, que se imparte en el primer semestre del $2^{\circ}$ curso del Grado en Medicina (www.uspceu.com). Dicha asignatura es de carácter obligatorio y consta de 7,5 créditos ECTS. La experiencia docente se ha llevado a cabo de forma simultánea en los cuatro grupos lo que supone un total de 148 alumnos matriculados.

\section{Técnicas, instrumentos y recursos utilizados}

Para llevar a cabo esta experiencia docente se ha empleado la plataforma BlackBoard (vía intranet), que permite el desarrollo de forma integral de la enseñanza y aprendizaje en línea (on-line). Esta plataforma frece una gran variedad de recursos que permiten impartir y distribuir contenidos en distintos formatos (texto, audio, vídeo y animación), realizar evaluaciones en línea, llevar a cabo el seguimiento académico de los alumnos participantes, originar tareas y desarrollar actividades en ambientes colaborativos.

\section{Metodología de trabajo}

Organización de los grupos de clase para la elaboración del vídeo

$\mathrm{Al}$ inicio del curso cada grupo de $2^{\circ}$ de Medicina se dividió en tres equipos. Cada equipo se encargó de estudiar y desarrollar un tema de los tres que fueron propuestos por el profesorado y que forman parte del programa de la asignatura. Los temas a elegir fueron: "Adaptaciones cardiorrespiratorias a las altas presiones", "Adaptaciones 
Experiencia en la elaboración de vídeos didácticos por alumnos de Fisiología como parte de su proceso de aprendizaje.

cardiorrespiratorias a las bajas presiones" y "Adaptaciones cardiorrespiratorias al ejercicio". Los alumnos podían elegir de forma voluntaria en qué equipo querían participar. Los tres grupos en los que se dividió la clase tuvieron aproximadamente el mismo número de alumnos. Por tanto, dado que había cuatro grupos y cada grupo estaba dividido en tres equipos, hubo un total de 12 equipos compuestos cada uno de 12 ó 13 alumnos. De este modo, cada equipo debía preparar fuera del aula una presentación en formato vídeo con los contenidos del tema que tenía asignado.

Creación de un foro como herramienta de comunicación para la elaboración del vídeo. A cada equipo se le creó en el campus virtual de la plataforma Blackboard un foro en el que sólo estaban incluidos los alumnos pertenecientes a ese equipo y los profesores de la asignatura. Dicho foro se creó como canal de comunicación entre los propios alumnos y entre los alumnos y el profesor, para preparar de forma eficaz y autónoma parte del temario de la asignatura, que luego debería ser presentado al resto de la clase en formato vídeo (Figura 1).

En la Figura 1 se pueden ver los foros creados en el campus virtual.

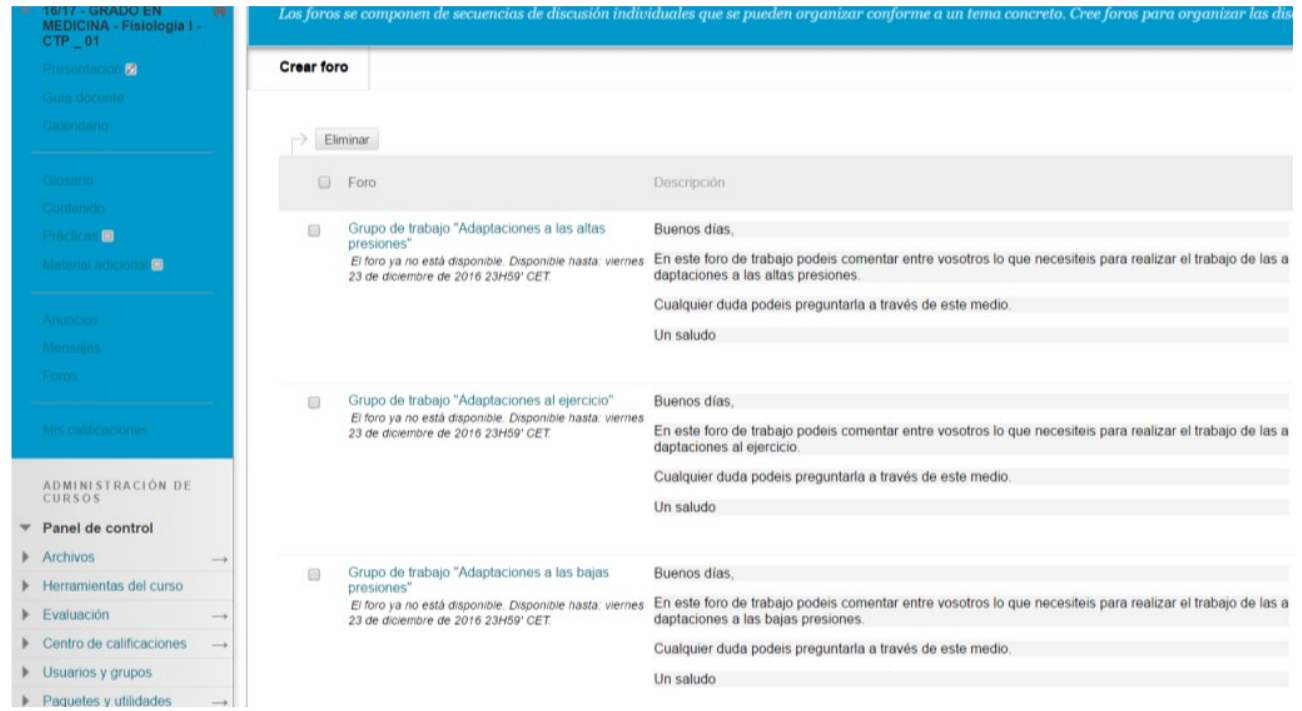

Figura 1. Creación del foro en el Campus Virtual.

\section{Indicaciones para la realización del vídeo.}

Cada equipo podía elegir qué contenidos querían incluir en el vídeo, es decir, tenían total libertad para desarrollarlo como quisieran y hablar de los aspectos que ellos consideraran oportunos. Solo se le dieron dos indicaciones:

- El vídeo debía tener una duración máxima de 10 minutos. Si se excedían en dicho tiempo no se admitirían para su evaluación.

- Todo lo relativo a la preparación del vídeo, dudas que iban surgiendo así como discusiones relativas al contenido debían ser expuestas en el foro.

(cc) EY-NC-ND 2017, Universitat Politècnica de València 


\section{Tutorización del trabajo}

A mitad del semestre se hizo una tutoría académica en la que los alumnos tenían que enseñar a los profesores el desarrollo de su trabajo, con el fin de orientarles y comprobar cómo lo estaban llevando a cabo. De forma continuada los profesores tutelaron el foro, de modo que el contenido que los alumnos iban subiendo era supervisado y se les iban haciendo las sugerencias oportunas, con el fin de optimizar su trabajo y que no incluyeran aspectos poco relevantes o erróneos.

Visualización de los vídeos.

Al final del semestre se dedicaron tres días a la proyección de los vídeos. Cada día se vieron los 4 vídeos del mismo tema elegido (uno por cada grupo del curso). Al finalizar la clase se hizo una votación y se les pidió a todos los alumnos que escribieran en una hoja el título del vídeo de cada tema que más les había gustado. Por otra parte, los profesores de la asignatura se reunieron para volver a visualizar los vídeos y evaluar fundamentalmente el contenido, pero también la edición del vídeo, originalidad, creatividad, participación e implicación de cada alumno.

Aunque en un principio no estaba previsto y la experiencia se desarrolló como una actividad obligatoria dentro de la asignatura, dada la buena acogida que hubo por parte de los alumnos se decidió organizarlo a modo de concurso, de manera que según la votación de los alumnos y de los profesores se otorgaron unos diplomas a los tres vídeos más votados de entre los doce.

\section{Análisis de la experiencia}

Una vez terminada la experiencia docente se elaboró una encuesta en la que los alumnos valoraron el desarrollo de la misma. Las preguntas que se les formularon fueron las siguientes:

1. Valore del 1 al 10 el esfuerzo que le ha supuesto la realización del vídeo para el trabajo de las adaptaciones (1 nada esfuerzo; 10 muchísimo esfuerzo)

2. Marque con una $X$ lo que ha supuesto para usted la realización del vídeo:

- Me ha supuesto mucho esfuerzo y no me ha servido absolutamente para nada.

- Me ha supuesto mucho esfuerzo pero me han servido para aprender.

- Me ha supuesto el mismo esfuerzo que cualquier otra actividad pero no he aprendido.

- Me ha supuesto el mismo esfuerzo que cualquier otra actividad y me ha servido para aprender de forma divertida.

- Me ha supuesto el mismo esfuerzo que cualquier otra actividad y me ha servido para aprender de forma aburrida.

- $\quad$ No me ha supuesto esfuerzo pero tampoco he aprendido.

- $\quad$ No me ha supuesto esfuerzo y he aprendido.

3. ¿Cree que es bueno que se siga haciendo esta actividad para futuros alumnos? Marque $\mathrm{Si} / \mathrm{No}$ 
Experiencia en la elaboración de vídeos didácticos por alumnos de Fisiología como parte de su proceso de aprendizaje.

4. Escriba a continuación si tiene alguna sugerencia/recomendación acerca de la realización del vídeo.

\section{Resultados}

\section{Valoración por parte de los profesores respecto a la elaboración de los vídeos.}

En la elaboración del vídeo los profesores valoramos dos aspectos:

En primer lugar el contenido académico de los mismos. En este sentido todos los vídeos mostrados tuvieron una elevada calidad en cuanto a su contenido. El abordaje del tema fue muy adecuado y los contenidos que se mostraron fueron precisos y de calidad científica. No hay que olvidar que los alumnos tenían plena libertad para incluir en él lo que consideraran más oportuno ya que solamente se les había dado el título del tema a desarrollar. Este hecho hizo que los distintos vídeos elaborados fueran muy diferentes, aún entre los que trataban del mismo tema, lo que hace más enriquecedora la experiencia ya que con la visualización de todos los vídeos se abordan más aspectos dentro de un mismo tema. De hecho, nos sorprendieron muy gratamente algunas ideas que se incluyeron en los vídeos, lo que pone de manifiesto que con el desarrollo de esta experiencia se potencian otras habilidades de los alumnos, como la creatividad, la originalidad y la innovación, además potenciar un espíritu crítico y riguroso.

En segundo lugar también quisimos valorar la edición de los vídeos. Tratándose de alumnos que estudian Medicina y, por tanto, no tendrían porqué estar familiarizados con estas herramientas, queremos resaltar que de los doce vídeos que se presentaron nueve de ellos tuvieron un formato excelente. Este hecho pone de manifiesto que la mayor parte de los alumnos están acostumbrados a utilizar esta herramienta de forma cotidiana por lo que no les resulta complicado utilizarlas como recurso para su aprendizaje. En el caso de los alumnos que tuvieron más dificultades tecnológicas éstos fueron capaces de resolver los problemas que les fueron surgiendo y así adquirieron una nueva habilidad para el aprendizaje.

\section{Valoración por parte de los alumnos respecto a la elaboración de los vídeos.}

Los resultados de las encuestas se muestran a continuación. En la Figura 2 se muestra el esfuerzo percibido por los alumnos respecto al desarrollo de esta actividad. Como se puede ver en una escala del 1 al 10, la mayor parte de los alumnos consideraron que la elaboración de los vídeos les ha supuesto un esfuerzo medio alto (puntuación 7-8) y sólo una pequeña parte de los alumnos consideraron que les ha supuesto mucho o poco esfuerzo. 


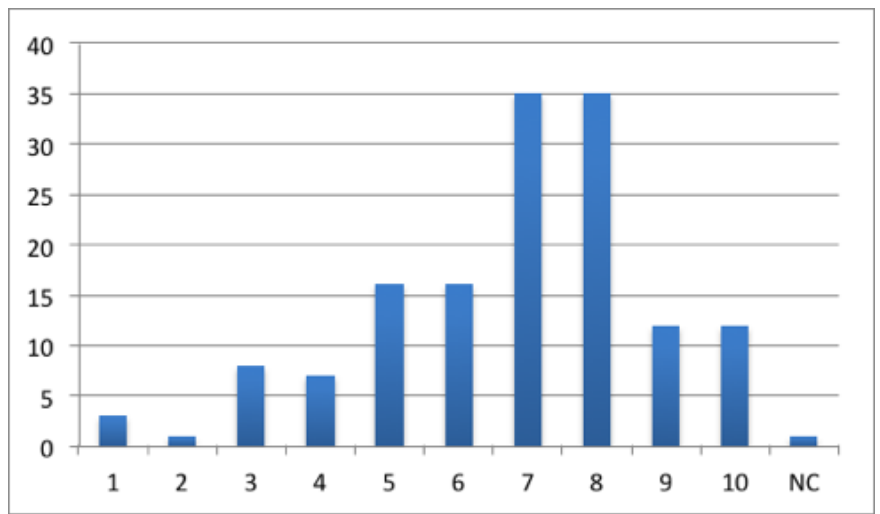

Figura 2. Esfuerzo percibido por los alumnos al realizar esta actividad

Respecto a la pregunta en la que se les consulta si creen que se debe seguir haciendo esta actividad para futuros alumnos, la respuesta ha sido mayoritariamente afirmativa (Figura 3).

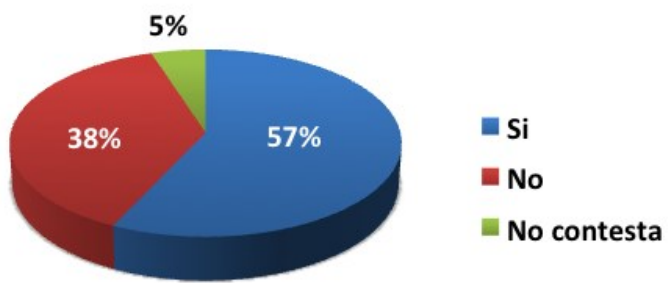

Figura 3. Resultados de la encuesta acerca de si se debe seguir realizando esta actividad.

Por último, quisimos saber si los alumnos consideraban positiva esta actividad en cuanto a su aprendizaje. Como se puede ver en la Figura 4, un $61 \%$ de todos los alumnos consideran que sí han aprendido al realizar el vídeo.

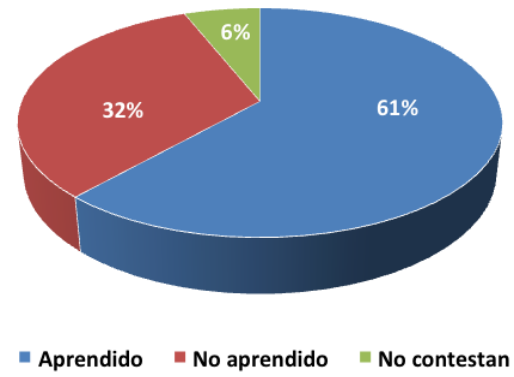

Figura 4. Percepción de los alumnos sobre su aprendizaje. 
Experiencia en la elaboración de vídeos didácticos por alumnos de Fisiología como parte de su proceso de aprendizaje.

Para finalizar, entre las sugerencias de los alumnos para mejorar esta actividad citaremos a continuación las más relevantes. Entre los comentarios de los alumnos queremos destacar la opinión de que es un buen método para aprender y que fomenta el trabajo en grupo. Como sugerencia de mejora una gran mayoría de los alumnos propuso que los grupos fueran más pequeños, es decir, con un número más reducido de alumnos.

\section{Resultados respecto al uso del foro}

Con el foro se pone a disposición del alumno una herramienta on-line que les permite "estar conectados" entre ellos y con el profesor de modo que tienen un "canal oficial" de comunicación y no tienen que buscar otras alternativas que suelen usar más a menudo como Google, Ask o WhatsApp. El foro se encuentra disponible en la plataforma del estudiante teniendo un acceso fácil y ágil al mismo. Por otro lado el profesor puede ver el trabajo que realizan sus alumnos, por lo que es capaz de discernir entre aquellos alumnos que participan en el foro y los que no, pudiendo incentivar la participación en el mismo. Además, a través de la participación del profesor se pueden resolver dudas, se les puede guiar, orientar y tutelar el trabajo.

Tras analizar el número de participaciones de los alumnos en el mismo y realizando el análisis sobre el conjunto de la totalidad de los alumnos que están matriculados en la asignatura, hemos encontrado los datos que mostramos a continuación.

La participación de los alumnos ha sido del $11 \%$ respecto al número de alumnos en total que han participado en el foro: alumnos totales de los cuatro grupos (Figura 5).

\section{\% participación}

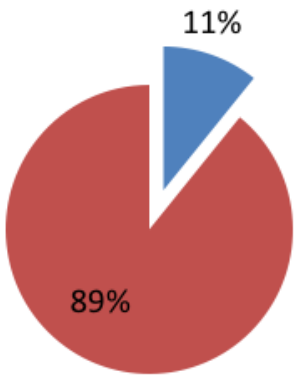

Figura 5. Porcentaje de participación en el foro de los 148 alumnos matriculados

Analizando los resultados observamos un porcentaje de participación en los foros bajo. Debemos tener en cuenta que no se han contabilizado a la hora de valorar a los participantes ni las entradas de los profesores ni las de los alumnos que han utilizado el foro repetidamente. 
Consideramos que la baja participación en el foro durante este curso se ha podido deber a varios motivos:

- Los alumnos no están familiarizados aún con el foro al ser la primera asignatura de su carrera que les ofrece esta posibilidad.

- Los alumnos, a pesar de que se les explicó, no han utilizado el foro para dialogar entre ellos (excepto en un grupo que si lo ha hecho), si no para preguntar dudas al profesor y colgar información.

- La interferencia de otras vías de comunicación con la que los alumnos están más familiarizados (WhatsApp) puede estar dificultando la participación en el foro.

Analizando los datos por sexos (Figura 6) observamos que el porcentaje de mujeres que han participado en la experiencia docente respecto al total de alumnos es de un $46 \%$ versus un $54 \%$ de hombres, no existiendo diferencias estadísticamente significativas en este aspecto. Sin embargo, hay que aclarar que del total de 148 alumnnos, un $71 \%$ se corresponde con mujeres y un $29 \%$ son hombres, por lo que si tenemos en cuenta estos datos cabría decir que los hombres han tenido una mayor participación en el uso del foro.

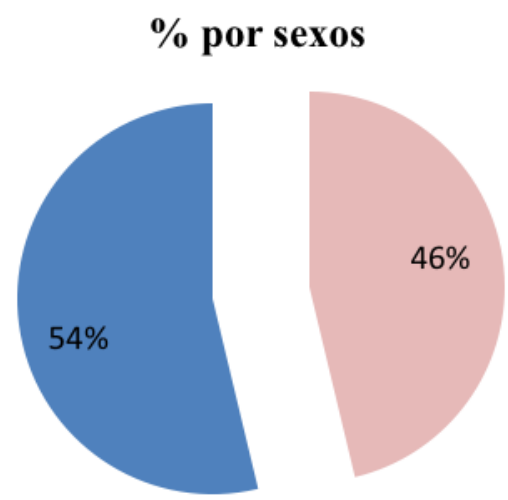

Figura 6. Participación por sexos en el foro teniendo en cuenta la totalidad de alumnos de segundo curso de Medicina

A pesar de que los porcentajes de participación no hayan sido elevados los profesores lo valoramos de manera positiva. Como se ha referido previamente, un grupo si lo utilizó como chat de la asignatura y fue muy enriquecedor, lo que nos anima a mantener el foro en los próximos cursos.

Se adjunta a continuación un ejemplo del uso de la plataforma como foro (Figura 7). 
Experiencia en la elaboración de vídeos didácticos por alumnos de Fisiología como parte de su proceso de aprendizaje.

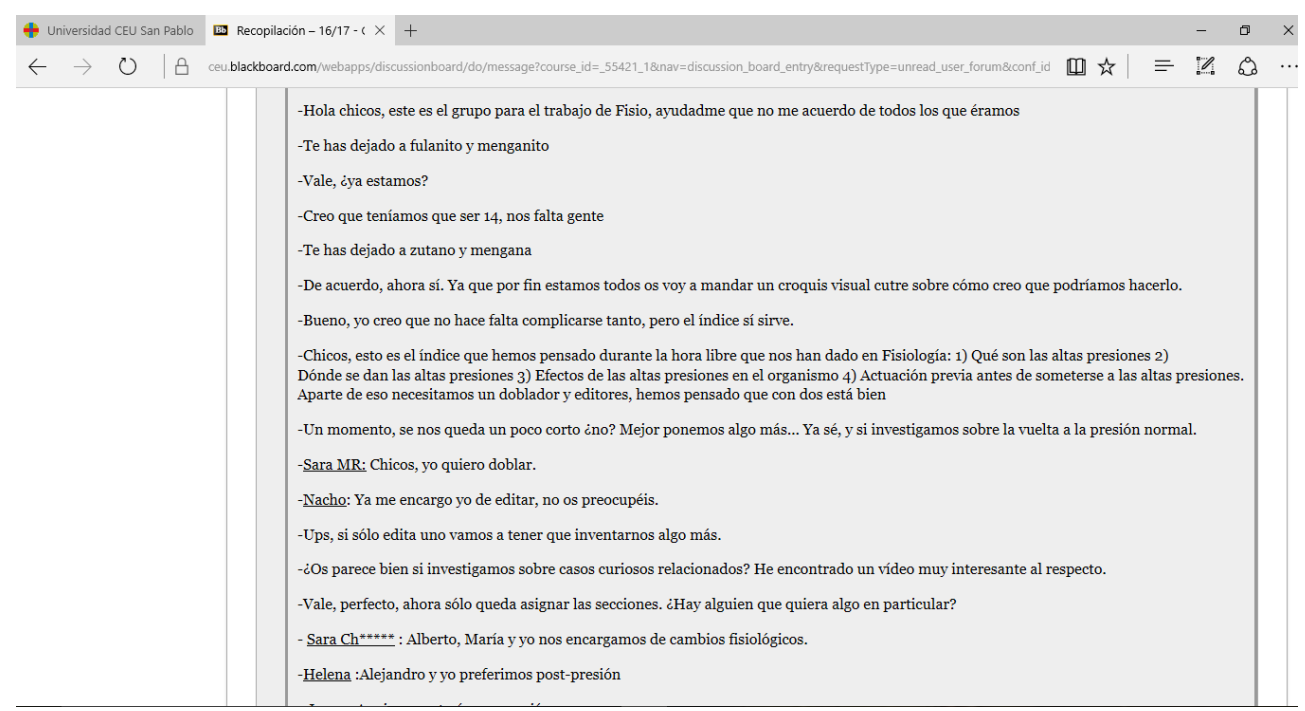

Figura 7. Ejemplo del uso del foro.

\section{Conclusiones}

Consideramos que la elaboración de un vídeo didáctico por los alumnos es una herramienta válida para favorecer un sistema de aprendizaje más activo y participativo por su parte. No sólo les permiten alcanzar los conocimientos de Fisiología, sino que también les ayuda a formarse en el uso de nuevas tecnologías como instrumento laboral, les ayuda a comprender la racionalización de los tiempos y a relacionarse entre ellos en un ambiente colaborativo, competencias todas ellas que les serán demandadas cuando se sumerjan en la vida profesional.

A pesar de la escasa participación de los alumnos en el uso del foro creemos que es una herramienta eficaz para tutelar el trabajo autónomo del alumno y para incrementar la comunicación entre profesores y alumnos y la de los alumnos entre ellos. Atribuimos su escasa participación al hecho de que es la primera vez que se les ofrece esta herramienta. Nos planteamos de cara al futuro nombrar un alumno voluntario que funcione como líder y se encargue de moderar el foro para mantenerlo en actividad sin depender tanto de la participación del profesor. Pensamos que así los alumnos lo puedan entender más como una sala propia de discusión en la que aportar ideas, dudas y conocimiento y en la que ellos sean los principales protagonistas.

En definitiva, como conclusiones finales podemos decir que el uso de herramientas docentes basadas en las nuevas tecnologías tiene una buena aceptación por parte de los alumnos, les motiva y les hace más participativos. Con la elaboración de vídeos didácticos los alumnos aprenden nuevos contenidos a la vez que se potencia su creatividad y se divierten. El proceso de diseño y desarrollo de un vídeo les estimula para

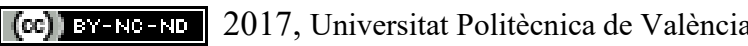


aprender de forma autónoma, saber discernir entre los conceptos que son más importantes, buscar información contrastada, colaborar entre ellos y trabajar en equipo.

\section{Referencias}

ARCEO FDB, ROJAS GH, GONZÁLEZ ELG (2002). Estrategias docentes para un aprendizaje significativo: una interpretación constructivista. Libro electrónico: mapas.eafit.edu.co.

ÁLVAREZ B, GONZÁLEZ MIERES C, GARCÍA RODRÍGUEZ N (2008). La motivación y los métodos de evaluación como variables fundamentales para estimular el aprendizaje autónomo. Revista de Educación Universitaria 1.

BARR, R.B Y TAGG, J. (1995). "From teaching to learning. A new paradigm for undergraduate education", en Change, 27 (6), pp. 13-25. Revista electrónica de Tecnología Educativa, $\mathrm{n}^{\circ} 29$

CASTAÑO, C. Y ROMERO, R. (2007): Las TIC en los procesos de formación. Nuevos medios, nuevos escenarios para la formación. En Cabero, J. y Romero, R. (coordinadores): Diseño y Producción de TIC para la formación. Barcelona: UOC.

FERRÉS, J. Y BARTOLOMÉ, A. (1991). EL VÍDEO, enseñar vídeo, enseñar con el vídeo. Barcelona: Gustavo Gili, S.A.

FERRO SOTO C, MARTÍNEZ SENRA AI, OTERO NEIRA MC (2009). Ventajas del uso de las TICs en el proceso enseñanza-aprendizaje desde la óptica de los docentes universitarios españoles.

GARCÍA VALCÁRCEL, A. (1993). "Análisis de los modelos de enseñanza empleados en el ámbito universitario", en Revista Española de Pedagogía, 194, 27-53.

GARGALLO, B, GARCÍA- FÉLIX, I, MORERA, C. Y BENAVENT, A. (2015). Métodos innovadores y enfoques de aprendizaje en estudiantes universitarios.

Doi:http://dx.doi.org/10.4995/INRED2015.2015.1576

MACHEMER, P. L. . Y CRAWFORD, P. (2007). "Student perceptions of active learning in a large crossdisciplinary classroom", en Active Learning in Higher Education, 8 (1), 930 .

MOLERO LÓPEZ-BARJAS D., RUIZ CARRASCOSA J (2005). La evaluación de la docencia universitaria. Dimensiones y variables más relevantes. Revista de Investigación Educativa 23 (1). 
Experiencia en la elaboración de vídeos didácticos por alumnos de Fisiología como parte de su proceso de aprendizaje.

MONEREO C. Y POZO J. I. (2003). La universidad ante la nueva cultura educativa, Enseñar y aprender para la autonomía. Madrid: Síntesis.

ROMÁN, P. Y LLORENTE, M (2007). El diseño de vídeos educativos: el vídeo digital. En Cabero, J. y Romero, R. (coordinadores): Diseño y Producción de TIC para la formación. Barcelona: UOC.

RUTH S. CONTRERAS ESPINOSA Y JOSE LUIS EGUIA (2016): Gamificación en aulas universitarias. Bellaterra : Institut de la Comunicació, Universitat Autònoma de Barcelona. ISBN 978-84-944171-6-0. 